\title{
Efficient Markov perfect Nash equilibria: theory and application to dynamic fishery games
}

\author{
G. Martín-Herrán, J.P. Rincón-Zapatero* \\ Departamento de Economía Aplicada, Facultad de Ciencias Económicas, Universidad de Valladolid, Avda. \\ Valle Esgueva 6, 47011 Valladolid, Spain
}

\begin{abstract}
In this paper, we present a method for the characterization of Markov perfect Nash equilibria being Pareto efficient in non-linear differential games. For that purpose, we use a new method for computing Nash equilibria with Markov strategies by means of a system of quasilinear partial differential equations. We apply the necessary and sufficient conditions derived to characterize efficient Markov perfect Nash equilibria to dynamic fishery games.
\end{abstract}

JEL classification: $\mathrm{C} 73 ; \mathrm{Q} 22$

Keywords: Differential games; Markov perfect Nash equilibria; Pareto optimum; Quasilinear partial differential equations; Fishery management

\section{Introduction}

The interdependence between the decisions of the agents is well known in many areas of economics. Game theory is a useful tool to study these situations involving several decision makers. An interesting question is to determine whether the non cooperative solution presents the property of Pareto efficiency. The question is

\footnotetext{
*Corresponding author. Tel.: + 34-983 186 591; fax: +34-983 423299.

E-mail address: zapatero@eco.uva.es (J.P. Rincón-Zapatero).
} 
whether a cooperative behavior can emerge without any binding agreements between the agents. The efficiency of Nash equilibrium is a very uncommon feature, but at the same time a very desirable property, since it makes the efficient solution selfenforcing. If, moreover, the Nash equilibrium is subgame perfect, the efficient solution can be enforced for any initial condition during the course of the game.

If a priori efficiency is not at hand, a standard method to implement cooperative solutions by means of non-cooperative play is to construct efficient Markov perfect Nash equilibria (MPNE) based on trigger strategies, as in Haurie and Pohjola (1987) or Tolwinski et al. (1986). Within the limits of differential games theory, this approach presents technical problems because trigger strategies are in general discontinuous and needs the introduction of memory strategies for the players. These strategies are based on all past information of the game evolution to the current time, and as a consequence, this kind of strategies are non-Markovian. Another option is the use of incentive strategies, establishing the efficient solution as an incentive equilibrium (see, for example, Ehtamo and Hämäläinen, 1989, 1993; Jørgensen and Zaccour, 2001).

It is important to note that the attainment of the cooperative solution as a Nash equilibrium of a non-cooperative game will depend on what set of strategies is available to the players. For that reason, this paper proposes a new approach that allows to identify games where the MPNE based on Markov strategies is Pareto efficient. This new approach is based on the characterization of MPNE as solutions to a system of quasilinear partial differential equations. This system is obtained from the optimality conditions of the maximum principle. The quasilinear system is fully equivalent to the Hamilton-Jacobi-Bellman system, as pointed out in RincónZapatero et al. (1998). The former system characterizes MPNE directly, whereas the latter characterizes the value functions. However, a quasilinear system is much more amenable than a fully non-linear system of partial differential equations as the Hamilton-Jacobi-Bellman system.

To our knowledge, there are no necessary and sufficient conditions in the literature to be applied to general differential game models that allow to determine whether the feedback Nash equilibrium is or not a Pareto optimum. This paper is devoted to establish a method that allows us to identify the coefficient functions of a rather general differential game in order that the game possesses an efficient MPNE. A particular case, for differential games with unidimensional state and control variables and where each control is a smooth function of the state and time variable, has been studied in Rincón-Zapatero et al. (2000). The importance of the identification of equilibria being Pareto optimum relies on the stability properties of Nash's concept and on the efficiency of the cooperative solution. An extreme case of this type of solutions are the so called absolutely cooperative, which are studied, e.g., in Leitmann (1974).

It is well known that, in general, the use of Markov strategies in non-cooperative games prevents to attain efficient outcomes (see, for example, Dubey, 1986). In fact, the efficiency of Nash equilibrium can be considered a very rare property. However, such a property has been noticed to hold for some fishery games Chiarella et al. (1984). In this paper, the players are restricted to use open-loop strategies, so the 
Nash equilibrium is not subgame perfect. Our aim is to analyze whether the same property can be guaranteed when the players use Markov strategies. Our approach is based on a necessary condition established in Theorem 2, which constitutes an easily implementable test for checking if the efficiency of the MPNE is possible in a concrete differential game. Once we substitute the functional relationship in the coupled quasilinear system, we obtain an overdetermined system. The existence of solutions is then tested with a well-known compatibility condition developed in the theory of partial differential equations, as it is shown in Theorem 3. This is the content of our sufficiency result.

The outline of the paper is as follows. Section 2 is devoted to state a differential game in general form and some of its cooperative and non-cooperative solutions. In particular, MPNE and Pareto optima for the non-cooperative and cooperative games, respectively. A brief presentation of the new characterization of Nash equilibrium as solution to a system of quasilinear partial differential equations is also included. A more detailed analysis of this approach can be found in Rincón-Zapatero et al. (1998) and Martín-Herrán and Rincón-Zapatero (2002). In Section 3, Theorem 2, we establish a necessary condition for the Pareto efficiency of MPNE. In Theorem 3 , a set of sufficient conditions is given. In order that Nash equilibrium be a Pareto optimum, our approach uses a compatibility condition that makes the existence of common solutions of several partial differential equations possible. Let us observe that the compatibility condition is easier to be applied to a quasilinear partial differential equations system than to the Hamilton-Jacobi-Bellman system. The results are shown with three different economic differential games belonging to the class of dynamic fishery games in Section 4. Conclusions are presented in Section 5.

\section{Description of the game: characterization of MPNE}

In this section, we present the characteristics of the differential games considered along the paper, some definitions and the results we will use in the following sections. First we introduce some notation. A subscript indicates partial differentiation; the partial derivative of a scalar function with respect to a vector and the partial derivative of a vector function with respect to a scalar are defined as column vectors. Also, the partial derivative of a vector function with respect to another vector is defined as a matrix, e.g. $h_{z}=\partial h / \partial z=\left(\partial h^{i} / \partial z^{j}\right)_{n \times m}$, where $h$ and $z$ are $n \times 1$ and $m \times 1$ vectors, respectively. The superscript $T$ denotes the transposition sign.

We consider an $N$-person differential game over a fixed and bounded time interval $[0, T]$. The formulation of the game for the infinite horizon case is quite similar, the only difference being that there is no bequest function for the players. Please see below for the definition of the bequest function.

For $i=1, \ldots, N$, player $i$ chooses a vector of strategies $u^{i}=\left(u_{1}^{i}, \ldots, u_{n}^{i}\right)^{\top} \in U^{i}$ to influence the vector of state variables, $y=\left(y^{1}, \ldots, y^{n}\right)^{\top} \in \mathbb{R}^{n}$, which evolution is given by the system of ordinary differential equations

$$
\dot{y}(s)=f\left(s, y(s), u^{1}(s), \ldots, u^{N}(s)\right), \quad t \leqslant s \leqslant T,
$$


with initial condition

$$
y(t)=x, \quad t \in[0, T], \quad x \in \mathbb{R}^{n},
$$

and where the pair $(t, x)$ is the root from which the dynamic game proceeds. Since our aim is to work with the MPNE concept, we need to consider the game for every $\operatorname{root}(t, x) . U^{i} \subseteq \mathbb{R}^{n}$ denotes the control region of $i$ th player.

Definition 1 (Admissible strategies). A strategic profile $u=\left(u^{1}, \ldots, u^{N}\right)$ is called admissible if $u(s) \in U^{1} \times \cdots \times U^{N}$ for every $s \in[0, T]$ and

(i) for every $(t, x)$ the system (1) with initial condition $y(t)=x$ admits a unique solution;

(ii) for each $i=1, \ldots, N$, there exists some function $\phi^{i}:[0, T] \times \mathbb{R}^{n} \longrightarrow U^{i}$ of class $\mathscr{C}^{1}$ such that $u^{i}(s)=\phi^{i}(s, y(s))$ for every $s \in[0, T]$.

Let $\mathscr{U}^{i}$ denote the set of admissible strategies of player $i$ and $\mathscr{U}=\mathscr{U}^{1} \times \cdots \times \mathscr{U}^{N}$ the set of admissible strategy profiles. Notice that we are considering Markov controls for the players. If $\phi^{i}$ is time independent, the corresponding control is called a stationary Markov control. Sometimes we will identify $u^{i}$ and $\phi^{i}$ in the notation.

The instantaneous utility function of player $i$ is denoted by $L^{i}$ and his or her bequest function by $S^{i}$. Given $(t, x) \in[0, T] \times \mathbb{R}^{n}$ and an admissible strategic profile $u$, the payoff functional of each player is given by

$$
J^{i}(t, x ; u)=\int_{t}^{T} \mathrm{e}^{\rho_{i}(s t)} L^{i}(s, y(s), u(s)) \mathrm{d} s+\mathrm{e}^{\rho_{i}(T}{ }^{t)} S^{i}(T, y(T)),
$$

with $\rho_{i} \geqslant 0$ the discount rate. The functions

$$
\begin{aligned}
& f:[0, T] \times \mathbb{R}^{n} \times U^{1} \times \cdots \times U^{N} \longrightarrow \mathbb{R}^{n}, \\
& L^{i}:[0, T] \times \mathbb{R}^{n} \times U^{1} \times \cdots \times U^{N} \longrightarrow \mathbb{R}, \\
& S^{i}:[0, T] \times \mathbb{R}^{n} \longrightarrow \mathbb{R},
\end{aligned}
$$

are all assumed to be of class $\mathscr{C}^{1} . J^{i}(t, x ; u)$ denotes the utility obtained by player $i$ when the games starts at $(t, x)$ and the profile of strategies is $u$.

In the infinite horizon case, which is important for us since the worked examples in the paper belong to this class of games, the bequest function is of course null. In this case, if the problem is autonomous and the strategies are Markov stationary, the value function is independent of time, and the initial condition is simply $x$, with $t=0$.

In a non-cooperative setting the aim of the players is to maximize their individual payoff $J^{i}$. Since this aspiration depends on the strategies selected by the other players also, it is generally impossible to attain. An adequate concept of solution is Nash equilibrium, which prevents unilateral deviations of the players from its recommendation of play. 
Definition $2(M P N E)$. An $N$-tuple of strategies $\widehat{\phi} \in \mathscr{U}$ is called a Markov perfect Nash equilibrium if for every $(t, x) \in[0, T] \times \mathbb{R}^{n}$, for every $\phi^{i} \in \mathscr{U}^{i}$

$$
J^{i}\left(t, x ;\left(\phi^{i} \mid \widehat{\phi}_{i}\right)\right) \leqslant J^{i}(t, x ; \widehat{\phi}),
$$

for all $i=1, \ldots, N$.

In the definition $\left(\phi^{i} \mid \widehat{\phi}_{i}\right)$ denotes $\left(\widehat{\phi}^{1}, \ldots, \widehat{\phi}^{i}{ }^{1}, \phi^{i}, \widehat{\phi}^{i+1}, \ldots, \widehat{\phi}^{N}\right)$. Note that with a MPNE no player has incentives to deviate unilaterally from the equilibrium, whatever the initial condition $(t, x)$ is.

On the other hand, if the game is cooperative, the players can coordinate their strategies in order to attain a better total payoff. An $N$-tuple of strategies is Paretooptimal if adopting another strategic profile, either the payoff of each player does not change or at least the payoff of one player decreases. So that, in both cases there is no reason for adopting another profile of strategies.

Definition 3 (Pareto optimality). An strategic profile $\widetilde{\phi}=\left(\widetilde{\phi}^{1}, \ldots, \widetilde{\phi}^{N}\right)^{\top}$ is called Pareto-optimal for every initial condition $(t, x)$, if for any other strategic profile $\phi=\left(\phi^{1}, \ldots, \phi^{N}\right)^{\top}$, either

$$
J^{i}(t, x ; \phi)=J^{i}(t, x ; \widetilde{\phi})
$$

for all $i=1, \ldots, N$, or there is at least one $i \in\{1, \ldots, N\}$ such that

$$
J^{i}(t, x ; \phi)<J^{i}(t, x ; \widetilde{\phi}) \text {. }
$$

We define the Hamiltonian function $H^{i}\left(t, x, u, \mu^{i}\right)=L^{i}(t, x, u)+f^{\top}(t, x, u) \mu^{i}$, where $\mu^{i}=\left(\mu_{1}^{i}, \ldots, \mu_{n}^{i}\right)^{\top}$ is the vector of costate variables associated to the $i$ th player.

Our aim is to establish useful conditions that allow us to determine the relationship between the coefficient functions defining the game, if any, assuring the Pareto-optimality of the MPNE. To this end, we will use the characterization of MPNE as a solution of a system of quasilinear partial differential equations, as was proposed in Rincón-Zapatero et al. (1998) and afterwards extended to the nonsmooth case in Rincón-Zapatero (2004). In the former paper the following was proved, by means of the maximum principle. If $\widehat{\phi} \in \mathscr{U}$ is a MPNE of the game, which is interior to the control region $U^{i} \times \cdots \times U^{N}$, then it must satisfy the following system of partial differential equations:

$$
\begin{aligned}
& \widehat{H}_{u^{i} t}^{i}+\widehat{H}_{u^{i} x}^{i} f+\widehat{H}_{u^{i} u}^{i}\left(\widehat{\phi}_{t}+\widehat{\phi}_{x} f\right)+\widehat{H}_{u^{i} \mu^{i}}^{i}\left(-\widehat{H}_{x}^{i}-\widehat{\phi}_{x}^{\top} \widehat{H}_{u}^{i}\right)=0, \\
& \quad \text { for } i=1, \ldots, N,
\end{aligned}
$$

where $\widehat{H}_{\{.\}}^{i}$ means $H_{\{\cdot\}}^{i}$ evaluated at $\left(t, x, \widehat{\phi}, \lambda^{i}\right)$. In $(4), \phi^{1}, \ldots, \phi^{N}$ are the dependent variables - the unknowns - and $t, x$ the independent variables. Since the Nash equilibrium is interior, the costate vectors that appear in the Hamiltonians associated with the players are substituted by means of the necessary condition of maximization of each player's Hamiltonian:

$$
\lambda^{i}=-f_{u^{i}}^{\top} L_{u^{i}}^{i} .
$$


This way we obtain system (4) of $n \times N$ quasilinear partial differential equations that must be satisfied for a MPNE of class $\mathscr{C}^{1}$.

The boundary condition over the costate variable established by the maximum principle and the expression obtained from the maximization of the Hamiltonian function provide a complete set of final conditions for the system (4) given by

$$
L_{u^{i}}^{i}(T, x, \widehat{\phi})+f_{u^{i}}^{\top}(T, x, \widehat{\phi}) S_{x}^{i}(T, x)=0 .
$$

In Rincón-Zapatero et al. (1998) we show that under suitable hypotheses about the Hamiltonian functions a $\mathscr{C}^{1}$ solution of (4), (6) becomes a MPNE of the differential game. That is, this system not only gives a set of necessary conditions but also sufficient for optimality. The sufficiency result can be summarized as follows:

Theorem 1. Let $\widehat{\phi} \in \mathscr{U}$ be a global $\mathscr{C}^{1}$ solution of (4), (6) interior to the control region and satisfying $\operatorname{det}\left|f_{u^{i}}(t, x, \widehat{\phi})\right| \neq 0$ for all $(t, x) \in[0, T] \times \mathbb{R}^{n}$, for all $i=1, \ldots, N$. Suppose further that for every $(t, x)$ and for all $\phi^{i} \in \mathscr{U}^{i}$

$$
H^{i}\left(t, x,\left(\phi^{i} \mid \widehat{\phi}_{i}\right), \Gamma^{i}\left(t, x, \widehat{\phi}^{i}\right)\right) \leqslant H^{i}\left(t, x, \widehat{\phi}, \Gamma^{i}\left(t, x, \widehat{\phi}^{i}\right)\right),
$$

for all $i=1, \ldots, N$. Then $V_{x}^{i}(t, x)=\Gamma^{i}(t, x, \widehat{\phi}(t, x))$ and $\widehat{\phi}$ is a MPNE of the game (1)-(3).

In the statement of the theorem, $\Gamma^{i}(t, x, u)=-\left(f_{u^{i}}^{\top} L_{u^{i}}^{i}\right)(t, x, u)$ and $V^{i}$ denotes the value function of the $i$ th player

$$
V^{i}(t, x)=\max _{\phi^{i} \in \mathscr{U}^{i}}\left\{J^{i}\left(t, x ;\left(\phi^{i} \mid \widehat{\phi}_{i}\right)\right): \dot{y}=f\left(s, y,\left(\phi^{i} \mid \widehat{\phi}_{i}\right)\right), y(t)=x, \forall s \in(t, T)\right\} .
$$

Remark 1. Theorem 1 states that the costate variables of the players coincide with the gradient of the value function with respect to $x, \lambda^{i}(t)=V_{x}^{i}(t, x)$.

Remark 2. In the infinite horizon case, suppose that $J^{i}(t, x ; u)$ converges uniformly for all admissible profile $u \in \mathscr{U}$. Let $\widehat{\phi}$ be a solution to (4) satisfying

$$
\lim _{T \rightarrow \infty} \Gamma^{i}(T, y(T), \widehat{\phi}(T, y(T))) \frac{\partial y}{\partial x}(T)=0,
$$

for every $i=1, \ldots, N$, where $(\partial y / \partial x)(T)$ denotes the derivative with respect to $x$ of the solution to (1)-(2) associated with $\widehat{\phi}$. Consider the solution $z$ of (1)-(2) associated to the strategic profile $\left(\phi^{i} \mid \widehat{\phi}_{i}\right)$, for an arbitrary $\phi^{i} \in \mathscr{U}^{i}$. Suppose further that

$$
\lim \sup V(T, z(T)) \geqslant 0 \text {. }
$$

Then it can be proved that $\widehat{\phi}$ is a MPNE of the infinite horizon game.

\section{Necessary and sufficient conditions for Pareto efficiency of MPNE}

In this section, we center our attention on the statement of necessary and sufficient conditions for Pareto efficiency of MPNE. The Nash equilibrium concept presents an important feature related to stability: no player can achieve a better result by 
deviating unilaterally from his or her Nash controls as long as the other players continue to use their Nash strategies. However, this solution can give inefficient outcomes. On the other hand, the players do not have incentives to play Pareto optima if they are not able to attain binding agreements.

The next result establishes that in order for a MPNE to be Pareto optimum it is necessary that no prisoners' dilemma occurs in the static game with payoff functions $\left(H^{1}, \ldots, H^{N}\right)$, for all $(t, x) \in[0, T] \times \mathbb{R}^{n}$. However, this is only a necessary condition. The Pareto optimality of Nash strategies in the static Hamiltonian game does not prevent the non-efficiency of Nash equilibria. This was already noted in Starr and Ho (1969).

Theorem 2. Let $\widehat{\phi} \in \mathscr{U}$ be a $\mathscr{C}^{1} M P N E$ of the game (1) (3), interior to the control region and satisfying $\operatorname{det}\left|f_{u^{i}}(t, x, \hat{\phi})\right| \neq 0$ for all $(t, x) \in[0, T] \times \mathbb{R}^{n}, i \in\{1, \ldots, N\}$. Let $H^{i}$ be of class $\mathscr{C}^{2}$ with respect to $u \in U$ for all $(t, x) \in[0, T] \times \mathbb{R}^{n}, i=1, \ldots, N$. Assume that the following set of conditions hold:

$$
\operatorname{sign}\left(H_{u^{j}}^{i}\left(t, x, \widehat{\phi}, \Gamma^{i}\right)\right)=\operatorname{sign}\left(H_{u^{j}}^{k}\left(t, x, \widehat{\phi}, \Gamma^{k}\right)\right),
$$

for all $i, j, k \in\{1, \ldots, N\}$ with $i, k \neq j$. If $\widehat{\phi}$ is Pareto optimum, then for all $(t, x) \in$ $[0, T] \times \mathbb{R}^{n}$ there exists $i \in\{1, \ldots, N\}$ such that for all $j \in\{1, \ldots, N\}, j \neq i$

$$
\left(f_{u^{j}}^{-\top} L_{u^{j}}^{i}-f_{u^{i}}^{-\top} L_{u^{i}}^{i}\right)(t, x, \widehat{\phi})=0 .
$$

Proof. For $i$ fixed let us suppose, by way of contradiction, that there exist $j \in$ $\{1, \ldots, N\}, i \neq j, \bar{t} \in[0, T]$ and $\bar{x} \in \mathbb{R}^{n}$ such that

$$
\left(f_{u^{j}}^{-\top} L_{u^{j}}^{i}-f_{u^{i}}^{-\top} L_{u^{i}}^{i}\right)(\bar{t}, \bar{x}, \widehat{\phi}) \neq 0 .
$$

Since $H^{i}$ is $\mathscr{C}^{2}$, for every $(t, x) \in[0, T] \times \mathbb{R}^{n}$ we have for all $u \in U$

$$
\begin{aligned}
H^{i}\left(t, x, u, \Gamma^{i}\right)= & H^{i}\left(t, x, \widehat{\phi}, \Gamma^{i}\right)+\sum_{k}^{N}\left(u^{k}-\widehat{\phi}^{k}\right) H_{u^{k}}^{i}\left(t, x, \widehat{\phi}, \Gamma^{i}\right) \\
& +\frac{1}{2} \sum_{k}^{N} \sum_{l=1}^{N}\left(u^{k}-\widehat{\phi}^{k}\right) H_{u^{k} u^{l}}^{i}\left(t, x, \bar{u}_{i}, \Gamma^{i}\right)\left(u^{l}-\widehat{\phi}^{l}\right)^{\top},
\end{aligned}
$$

with $\bar{u}_{i}$ in the segment $[\widehat{\phi}(t, x), u(t, x)]$. Furthermore, due to the subgame perfectness property of $\widehat{\phi}$ and the fact that it is interior to $U^{i}$ we have

$$
H_{u^{i}}^{i}\left(t, x, \widehat{\phi}, \Gamma^{i}\right)=0, \quad \text { for all }(t, x) \in[0, T] \times \mathbb{R}^{n} .
$$

Then, for $i \neq j$

$$
H_{u^{j}}^{i}\left(t, x, \widehat{\phi}, \Gamma^{i}\right)=\left(L_{u^{j}}^{i}-f_{u^{j}}^{\top} f_{u^{i}}^{-\top} L_{u^{i}}^{i}\right)(t, x, \widehat{\phi})=\left(f_{u^{j}}^{\top}\left(f_{u^{j}}^{-\top} L_{u^{j}}^{i}-f_{u^{i}}^{-\top} L_{u^{i}}^{i}\right)\right)(t, x, \widehat{\phi}),
$$

for all $(t, x) \in[0, T] \times \mathbb{R}^{n}$. As a consequence of hypothesis $(12), H_{u^{j}}^{i}\left(\bar{t}, \bar{x}, \widehat{\phi}, \Gamma^{i}\right) \neq 0$ and obviously, this inequality holds in a neighborhood $\mathcal{O}$ of $(\bar{t}, \bar{x})$.

For $\varepsilon \in \mathbb{R}^{n}$ with all components positive and sufficiently small, let us consider $\phi^{k}=\widehat{\phi}^{k}+\varepsilon S$, for all $k \in\{1, \ldots, N\}$, where $S$ denotes a $n \times n$ diagonal matrix, 
having each diagonal element equal to 1 if the corresponding component of $H_{u^{j}}^{i}\left(t, x, \widehat{\phi}, \Gamma^{i}\right)$ is positive and equal to -1 if it is negative. Then $\phi^{k} \in \mathscr{U}^{k}$ and the quadratic terms in the Taylor expansion are negligible with respect to the other terms. With this choice and taking into account (10) we have, for $\phi=\left(\phi^{1}, \ldots, \phi^{N}\right)$

$$
H^{i}\left(t, x, \phi, \Gamma^{i}\right) \geqslant H^{i}\left(t, x, \widehat{\phi}, \Gamma^{i}\right) \text { for every }(t, x) \in[0, T] \times \mathbb{R}^{n},
$$

with strict inequality in $\mathcal{O}$, as can be seen from (13). It has to be proved that $J^{i}(t, x ; \phi)>J^{i}(t, x ; \widehat{\phi})$ and $J^{k}(t, x ; \phi) \geqslant J^{k}(t, x ; \widehat{\phi})$ for $k \neq i$, in order to contradict that $\widehat{\phi}$ is a Pareto optimum. To this end, let $y$ be the solution of the differential equation

$$
\dot{y}(s)=f\left(s, y(s), \phi^{1}(s, y(s)), \ldots, \phi^{N}(s, y(s))\right), \quad y(\bar{t})=\bar{x}, \quad s \in[\bar{t}, T] .
$$

Denoting $u(s)=\phi(s, y(s))$ and $\widehat{u}(s)=\widehat{\phi}(s, y(s))$, substituting $t=s, x=y(s)$ and adding $J_{t}^{i}(s, y(s) ; \widehat{\phi})-\rho_{i} J^{i}(s, y(s) ; \widehat{\phi})$ to both sides of inequality (14) we have

$$
\begin{aligned}
& H^{i}\left(s, y(s), u(s), \Gamma^{i}(s, y(s), \widehat{u}(s))\right)+J_{t}^{i}(s, y(s) ; \widehat{\phi})-\rho_{i} J^{i}(s, y(s) ; \widehat{\phi}) \\
& \quad \geqslant H^{i}\left(s, y(s), \widehat{u}(s), \Gamma^{i}(s, y(s), \widehat{u}(s))\right)+J_{t}^{i}(s, y(s) ; \widehat{\phi})-\rho_{i} J^{i}(s, y(s) ; \widehat{\phi})
\end{aligned}
$$

for all $s \geqslant \bar{t}$, with strict inequality for all $s$ such that $(s, y(s)) \in \mathcal{O}$. If we derive the expression with respect to $s$

$$
J^{i}(s, y(s) ; \widehat{\phi})=\int_{s}^{T} \mathrm{e}^{\rho_{i}(\tau s)} L^{i}(\tau, y(\tau), \widehat{u}(\tau)) \mathrm{d} \tau+\mathrm{e}^{\rho_{i}(T s)} S^{i}(T, y(T)),
$$

we obtain

$$
J_{t}^{i}(s, y(s) ; \widehat{\phi})+J_{x}^{i}(s, y(s) ; \widehat{\phi}) f(s, y(s), \widehat{u}(s))=-L^{i}(s, y(s), \widehat{u}(s))+\rho_{i} J^{i}(s, y(s) ; \widehat{\phi}) .
$$

Taking into account Theorem $1, \Gamma^{i}(s, y(s), \widehat{u}(s))=V_{x}^{i}(s, y(s))=J_{x}^{i}(s, y(s) ; \widehat{\phi})$, hence rearranging terms from (16) we get

$$
H^{i}\left(s, y(s), \widehat{u}(s), \Gamma^{i}(s, y(s), \widehat{u}(s))\right)+J_{t}^{i}(s, y(s) ; \widehat{\phi})-\rho_{i} J^{i}(s, y(s) ; \widehat{\phi})=0
$$

and, in consequence, (15) implies

$$
H^{i}\left(s, y(s), u(s), \Gamma^{i}(s, y(s), \widehat{u}(s))\right)+J_{t}^{i}(s, y(s) ; \widehat{\phi})-\rho_{i} J^{i}(s, y(s) ; \widehat{\phi}) \geqslant 0,
$$

with strict inequality for $s$ such that $(s, y(s)) \in \mathcal{O}$. Rearranging terms again and multiplying by e ${ }^{\rho_{i}(s \bar{t})}$, we obtain

$$
\frac{\mathrm{d}}{\mathrm{d} s}\left(\mathrm{e}^{\rho_{i}(s \bar{t})} J^{i}(s, y(s) ; \widehat{\phi})\right)+\mathrm{e}^{\rho_{i}(s \bar{t})} L^{i}(s, y(s), u(s)) \geqslant 0 .
$$

Integrating the above inequality in $[\bar{t}, T]$ we have

$$
\mathrm{e}^{\rho_{i}\left(T^{\bar{t}}\right)} S^{i}(T, y(T))-J^{i}(\bar{t}, \bar{x} ; \widehat{\phi})+\int_{\bar{t}}^{T} \mathrm{e}^{\rho_{i}(s \quad \bar{t})} L^{i}(s, y(s), u(s)) \mathrm{d} s \geqslant 0,
$$

that is $J^{i}(\bar{t}, \bar{x} ; \phi)>J^{i}(\bar{t}, \bar{x} ; \widehat{\phi})$ and analogously for all $k \neq i, J^{k}(\bar{t}, \bar{x} ; \phi) \geqslant J^{k}(\bar{t}, \bar{x} ; \widehat{\phi})$, due to the selection made on $u^{k}$ in the Taylor expansion of each $H^{k}$. Consequently, the Nash equilibrium cannot be Pareto optimum. 
Remark 3. The main ingredients in the previous theorem are the existence of an interior and $\mathscr{C}^{1}$ MPNE and the identity $\Gamma^{i}(t, x, \widehat{\phi}(t, x))=V_{x}^{i}(t, x), i=1, \ldots, N$, which was rigorously proved in Rincón-Zapatero et al. (1998). The proof also works if the dimension of the state variable and the control variables are different but the two conditions just remarked hold.

Remark 4. The concavity of Hamiltonian $H^{i}$ with respect to variables $\left(u^{1}, \ldots, u^{N}\right)$ implies that the necessary condition (11) for the $i$ th player is also sufficient for Pareto efficiency of Nash equilibrium. This can be seen from the Taylor expansion (13), where the second order term is negative. In this case the MPNE is in fact the optimal profile that the $i$ th player would choose in case he or she could manage all the control variables. Hence, the efficient MPNE would arise as the optimal control profile when the $i$ th player has all the bargaining power in the cooperative game. If condition (11) is satisfied for all $i=1, \ldots, N$ together with the supplementary hypothesis of concave Hamiltonians $H^{i}, i=1, \ldots, N$, with respect to all the control variables, for all $(t, x) \in[0, T] \times \mathbb{R}^{n}$, then the MPNE is an absolutely cooperative solution. Let us note that condition (11) is fulfilled for zero-sum games and team problems.

Remark 5. The necessary condition (11) established in Theorem 2 for $N$-person games with unidimensional state and control variables can be written

$$
\left(L_{u^{j}}^{i} f_{u^{i}}-L_{u^{i}}^{i} f_{u^{j}}\right)(t, x, \widehat{\phi})=0, \quad \text { for all }(t, x) \in[0, T] \times \mathbb{R}^{n} .
$$

Remark 6. The hypothesis (10) can be eliminated in the statement of Theorem 2 when a two-person game is considered. The two-person game with a unique state variable and one control variable for each player was studied in Rincón-Zapatero et al. (2000).

Our purpose now is to establish sufficient conditions for Pareto efficiency of a MPNE. From now on, we concentrate on the two-person game with a unique state variable and one control variable for each player, but the following could be extended to a more general setting. We denote by $u^{2}=\varphi\left(t, x, u^{1}\right)$ the necessary relation (11) between the strategies of the two players. By replacing this relation in system (4) that holds for a MPNE, we obtain an overdetermined system. We look for conditions ensuring the existence of solutions to this system. To this end, we introduce the following notation for the two partial differential equations that arise from (4) once we have substituted $u^{2}=\varphi\left(t, x, u^{1}\right)$

$$
F^{i}\left(t, x, u^{1}, p, q\right)=a_{i}\left(t, x, u^{1}\right) p+b_{i}\left(t, x, u^{1}\right) q+c_{i}\left(t, x, u^{1}\right), \quad i=1,2 .
$$

Here $p=u_{t}^{1}, q=u_{x}^{1}$ and

$$
\begin{aligned}
a_{i} & =H_{u^{i} u^{i}}^{i}+H_{u^{i} u^{j}}^{i} \varphi_{u}, \\
b_{i} & =H_{u^{i} u^{i}}^{i} f+H_{u^{i} u^{j}}^{i} \varphi_{u} f-f_{u^{i}} H_{u^{j}}^{i} \varphi_{u}, \\
c_{i} & =H_{u^{i} t}^{i}+H_{u^{i} x}^{i} f+H_{u^{i} u^{j}}^{i}\left(\varphi_{t}+\varphi_{x} f\right)-f_{u^{i}}\left(H_{x}^{i}+H_{u^{j}}^{i} \varphi_{x}\right) .
\end{aligned}
$$

All functions are evaluated at $\left(t, x, u^{1}, \varphi\left(t, x, u^{1}\right)\right)$ and $\lambda^{i}=-f_{u^{i}}{ }^{1} L_{u^{i}}^{i}$. 
Assuming that $\Delta=\left|\begin{array}{l}a_{1} b_{1} \\ a_{2} b_{2}\end{array}\right| \neq 0$ for all $t, x, u^{1}$, then

$$
\left(\begin{array}{l}
p \\
q
\end{array}\right)=\left(\begin{array}{ll}
a_{1} & b_{1} \\
a_{2} & b_{2}
\end{array}\right)^{1}\left(\begin{array}{l}
-c_{1} \\
-c_{2}
\end{array}\right) \text {. }
$$

We denote by $\left[F^{1}, F^{2}\right]$ the following sum of determinants

$$
\left[F^{1}, F^{2}\right]=\left|\begin{array}{cc}
F_{x}^{1} & F_{q}^{1} \\
F_{x}^{2} & F_{q}^{2}
\end{array}\right|+p\left|\begin{array}{cc}
F_{u^{1}}^{1} & F_{p}^{1} \\
F_{u^{1}}^{2} & F_{p}^{2}
\end{array}\right|+\left|\begin{array}{cc}
F_{t}^{1} & F_{p}^{1} \\
F_{t}^{2} & F_{p}^{2}
\end{array}\right|+q\left|\begin{array}{cc}
F_{u^{1}}^{1} & F_{q}^{1} \\
F_{u^{1}}^{2} & F_{q}^{2}
\end{array}\right|,
$$

once we have replaced $p, q$ by their expressions given by (17).

Theorem 3. Suppose that the following conditions are satisfied:

(C1) The pair $\left(\widehat{\phi}^{1}, \varphi\right) \in \mathscr{U}$ is a $\mathscr{C}^{1}$ Markov Pareto optimum of problem (1)-(3), such that $f_{u^{1}}\left(t, x, \widehat{\phi}^{1}, \varphi\right) \neq 0$ for all $(t, x) \in[0, T] \times \mathbb{R}^{n}$.

(C2) $\left[F^{1}, F^{2}\right]=0$.

(C3) The pair $\left(\widehat{\phi}^{1}, \varphi\right)$ satisfies the final condition required in (6) associated to problem (1)-(3).

(C4) For all $u^{1} \in U^{1}, u^{2} \in U^{2}, t \in[0, T]$, for every $(t, x) \in[0, T] \times \mathbb{R}^{n}$

$$
\begin{aligned}
& H^{1}\left(t, x, \widehat{\phi}^{1}, \varphi, \Gamma^{1}\right) \geqslant H^{1}\left(t, x, \widehat{\phi}^{1}, u^{2}, \Gamma^{1}\right), \\
& H^{2}\left(t, x, \widehat{\phi}^{1}, \varphi, \Gamma^{2}\right) \geqslant H^{2}\left(t, x, u^{1}, \varphi, \Gamma^{2}\right),
\end{aligned}
$$

where $\Gamma^{i}$ equals $-f_{u^{i}}{ }^{1} L_{u^{i}}^{i}$ evaluated at $\left(t, x, \widehat{\phi}^{1}, \varphi\right), i=1,2$.

Then $\left(\widehat{\phi}^{1}, \varphi\right)$ is a MPNE.

Proof. When $\Delta \neq 0$, expression $\left[F^{1}, F^{2}\right]=0$ is a compatibility condition that ensures the existence of some common solution to the two partial differential equations, Ames (1965):

$$
\begin{aligned}
& F^{1}\left(t, x, u, u_{t}, u_{x}\right)=0 \\
& F^{2}\left(t, x, u, u_{t}, u_{x}\right)=0 .
\end{aligned}
$$

Since $F^{i}=0, i=1,2$ characterize the Nash equilibrium of the game and taking into account conditions (C3), and (C4), we can apply Theorem 1 and conclude that the pair $(\widehat{\phi}, \varphi)$ is a MPNE.

Remark 7. As we did in Remark 2, when an infinite horizon is considered, the final condition (C3) in Theorem 3 must be replaced by the sufficient transversality conditions (8) and (9).

Remark 8. When $\Delta \neq 0$ and the compatibility condition stated in Theorem 3 is not fulfilled, then the Pareto optimum cannot be a Nash equilibrium. In fact, $\left[F^{1}, F^{2}\right]=0$ is a necessary condition for the efficiency of Nash equilibrium. This provides a negative criterion based on a full computational method. When $\Delta=0$ and the equations $F^{1} \equiv 0, F^{2} \equiv 0$ are not such that one implies the other, then there is no common solution to the equations, hence again in this case the MPNE is not efficient. 


\section{Application to dynamic fishery games}

In this section the necessary and sufficient conditions for the efficiency of a MPNE established in Theorem 2 and in Theorem 3 are applied to dynamic fishery games. First a very general model of fishing borrowed from Chiarella et al. (1984) is stated. Let us consider $N$ countries that can access to $m$ fish populations. The instantaneous utility of the $i$ th country is

$$
L^{i}\left(c^{1}, \ldots, c^{N}, x_{1}, \ldots, x_{m}\right),
$$

where $x_{j}$ is the $j$ th fish population and $c^{i} \equiv\left(c^{i 1}, \ldots, c^{i m}\right)$ is the extraction vector of the $i$ th country, with $c^{i j}$ the extraction rate by the $i$ th country for the $j$ th fish population.

The population dynamics are described by the differential equations

$$
\dot{x}_{j}=G^{j}\left(c^{1}, \ldots, c^{N}, x_{1}, \ldots, x_{m}\right), \quad j=1, \ldots, m .
$$

This specification includes predator-prey and other types of biological interaction. The payoff functional of each player is given as in (3).

In Chiarella et al. (1984) four sets of conditions, under each of which there is an efficient Nash equilibrium path, are stated for some particular specifications of the fishery model described above. The solution concept used is open-loop. Although the efficiency of open-loop Nash equilibria for a class of fishery models is shown, by means of the necessary condition derived in Theorem 2 it can be proved that the Pareto optimal solution cannot be achieved as a MPNE. A similar result for a simple model of exploitation of a common-property non-renewable resource is stated in Dockner et al. (2000). The authors show that the achievement of the cooperative solution as a Nash equilibrium depends on what set of strategies is available to the players.

Let us consider that $L^{i}$ is a function of $c^{i}$ only. In Chiarella et al. (1984) it is proved that for this choice and independently of the population dynamics, there exists a Pareto-optimal open-loop Nash equilibrium.

Proposition 1. Assume that $L^{i}$ is a function of $c^{i}$ only, that for some component $c^{i j}$ there is no satiation point of $L^{i}$ and that

$$
G^{j}\left(c^{1}, \ldots, c^{N}, x_{1}, \ldots, x_{m}\right)=\widetilde{G}^{j}\left(x_{1}, \ldots, x_{m}\right)-\sum_{k}^{N} c^{k j} .
$$

Then the MPNE is not Pareto optimal.

Proof. Applying the necessary condition (11) for the specification given and for the $i$ th player we have

$$
L_{c^{i j}}^{i}=0, \quad \text { for all } j \in\{1, \ldots, N\},
$$

which is impossible for at least one $j$.

The condition $L_{c i j}^{i}=0$ means that the player maximizes instantaneously the utility function, without taking into account the dynamics of the species population. For that reason, this condition does not have sense for most of the utility functions used in the economic models, when a myopic behavior of the agents is ruled out. 
From now on we center on two different statements of a two species fishery differential game and we try to identify the coefficient functions of the games such that these admit efficient MPNE. The first example is taken from Clemhout and Wan (1985) who studied an infinite horizon differential game in the setting of a prey-predator system. The second example is borrowed from Hämäläinen et al. (1985). These authors analyze a finite horizon differential game in the framework of fishery management and in particular of the exploitation of the so-called transboundary fisheries.

Example 1. We consider the following two-person two-species differential game where players harvest from two different fish populations. Let us assume the following system dynamics:

$$
\begin{array}{ll}
\dot{x}_{1}=x_{1}\left(1-b_{11} \log x_{1}-b_{12} \log x_{2}\right)-c^{11}-c^{21}, & x_{1}(0)=x_{10}, \\
\dot{x}_{2}=x_{2}\left(1-b_{21} \log x_{1}-b_{22} \log x_{2}\right)-c^{12}-c^{22}, & x_{2}(0)=x_{20},
\end{array}
$$

where $x_{1}$ and $x_{2}$ denote the stocks of the two species and $c^{i j}$ denotes the harvest rate of player $i$ on species $j$. The payoff for the $i$ th player is given by

$$
W_{i}\left(x_{0}, c\right)=\int_{0}^{\infty} \mathrm{e}^{\rho_{i} t} g_{i}(x, c) \mathrm{d} t
$$

where $\rho_{i}$ is the discount rate and

$$
\begin{aligned}
& g_{i}(x, c)=\sum_{j}^{2}\left[w_{i j} \log x_{j}+\sum_{l 1}^{2} w_{i l j}^{c} \log c^{l j}\right], \\
& w_{i j} \geqslant 0, w_{i l j}^{c} \geqslant 0, w_{i j}^{c}>0, \quad \text { for all } i, j, l=1,2 .
\end{aligned}
$$

We have eliminated the time dependencies to shorten the notation. The differential game can be formulated as

$$
\max _{c^{i 1}, c^{i 2}}\left\{W_{i}\left(x_{0}, c\right): \quad \text { s.t. (18) (19) }\right\},
$$

for $i=1,2$.

In order to analyze the Pareto efficiency of MPNE we first focus our attention in the simplest specification: the two-person one-species differential game. The population dynamics for this game is

$$
\dot{x}=x(1-b \log x)-c^{1}-c^{2}, \quad x(0)=x_{0},
$$

where $x$ denotes the stock of the fish species and $c^{i}$ denotes the harvest rate of player $i$. The function $g_{i}(x, c)$ for the $i$ th player is

$$
\begin{aligned}
& g_{i}(x, c)=w_{i} \log x+\sum_{j}^{2} w_{i j}^{c} \log c^{j}, \\
& w_{i} \geqslant 0, w_{i j}^{c} \geqslant 0, w_{i i}^{c}>0, \quad \text { for all } i, j=1,2 .
\end{aligned}
$$


The necessary condition (11) for the first player implies

$$
c^{2}=\frac{w_{12}^{c}}{w_{11}^{c}} c^{1},
$$

establishing that the harvest rates of the players are proportional. Hence we must limit our search for efficient MPNE to strategic profiles fulfilling (20). System (4) satisfying the relationship between the strategies of the two players reads

$$
\begin{aligned}
& -c_{t}^{1} x+c_{x}^{1} x\left[c^{1} \frac{\left(w_{11}^{c}+w_{12}^{c}\right)}{w_{11}^{c}}-x(1-b \log x)\right] \\
& =-c^{1}\left[c^{1} \frac{w_{1}}{w_{11}^{c}}+x\left(1-b \log x-b-\rho_{1}\right)\right], \\
& -c_{t}^{1} x+c_{x}^{1} x\left[c^{1} \frac{w_{12}^{c}}{w_{11}^{c} w_{22}^{c}}\left(w_{21}^{c}+w_{22}^{c}\right)-x(1-b \log x)\right] \\
& =-c^{1}\left[c^{1} \frac{w_{12}^{c}}{w_{11}^{c} w_{22}^{c}} w_{2}+x\left(1-b \log x-b-\rho_{2}\right)\right] .
\end{aligned}
$$

Since we have stated an infinite horizon game and the problem is autonomous, stationary strategies are considered. In order to test if there is some solution to system (21)-(22) we make use of the compatibility condition (C2). For this game, it appears as a third degree homogeneous polynomial in the variables $x, c^{1}$, namely,

$$
a_{1}\left(c^{1}\right)^{3}+a_{2}\left(c^{1}\right)^{2} x+a_{3} c^{1} x^{2} .
$$

There are only two possible sets of conditions on the parameters under which the polynomial is identically null, and then the compatibility condition ensures the existence of at least one common solution to system (21)-(22).

- One-species game, I.

$$
\rho_{1}=\rho_{2}=\rho, \quad w_{12}^{c}=\frac{w_{1}}{w_{2}} w_{22}^{c}, \quad w_{21}^{c}=\frac{w_{2}}{w_{1}} w_{11}^{c}, \quad w_{1}>0, \quad w_{2}>0 .
$$

Under these conditions equations (21)-(22) are proportional, then it is sufficient to find a solution for, for example, the first one. It could be proved that the linear solution given by

$$
c^{1}=\frac{(b+\rho) w_{11}^{c} w_{2}}{w_{11}^{c} w_{2}+\left(w_{22}^{c}+w_{2}\right) w_{1}} x,
$$

satisfies Eq. (21), and therefore, Eq. (22). From condition (20) the corresponding harvest rate of the second player is

$$
c^{2}=\frac{(b+\rho) w_{22}^{c} w_{1}}{w_{11}^{c} w_{2}+\left(w_{22}^{c}+w_{2}\right) w_{1}} x .
$$


- One-species game, II.

$$
\rho_{1}=\rho_{2}=\rho, \quad w_{12}^{c}=\frac{\left(w_{1}+w_{11}^{c}\right) w_{22}^{c}}{w_{2}+w_{21}^{c}}, \quad w_{2}+w_{21}^{c}>0 .
$$

In this case these equations are not proportional and we have to look for solutions satisfying both equations simultaneously. It is easy to prove that the following linear solution satisfies system (21)-(22)

$$
c^{1}=\frac{(b+\rho) w_{11}^{c}\left(w_{2}+w_{21}^{c}\right)}{\left(w_{11}^{c}+w_{1}\right)\left(w_{22}^{c}+w_{2}+w_{21}^{c}\right)} x .
$$

As before, from condition (20) the corresponding harvest rate of the second player is

$$
c^{2}=\frac{(b+\rho) w_{12}^{c}\left(w_{2}+w_{21}^{c}\right)}{\left(w_{11}^{c}+w_{1}\right)\left(w_{22}^{c}+w_{2}+w_{21}^{c}\right)} x .
$$

In both cases, it is straightforward to show that the established strategy makes the dynamics stable if $b>0$.

The concavity of $H^{i}$ with respect to his or her own control variable is ensured and this property assures the fulfillment of condition (C4) in Theorem 3. Moreover, the Hamiltonians $H^{1}$ and $H^{2}$ are strictly concave with respect to all the control variables, which makes applicable Remark 4 guaranteeing that the necessary condition (11) is sufficient too and assuring the Pareto optimality of the pair $\left(c^{1},\left(w_{12}^{c} / w_{11}^{c}\right) c^{1}\right)$. Consequently, all the conditions listed in Theorem 3 are fulfilled.

Once we have characterized the efficient MPNE for the one-species game, summarized in Table 1, we return to the original two-species specification. The necessary condition (11) for the $i$ th player for this specification reads

$$
c^{2 j}=\frac{w_{i 2 j}^{c}}{w_{i 1 j}^{c}} c^{1 j}, \quad j=1,2,
$$

Table 1

Example 1. One species game

Efficient Markov perfect Nash equilibrium

Under (23):

$$
c^{1}=\frac{(b+\rho) w_{11}^{c} w_{2}}{w_{11}^{c} w_{2}+\left(w_{22}^{c}+w_{2}\right) w_{1}} x, \quad c^{2}=\frac{(b+\rho) w_{22}^{c} w_{1}}{w_{11}^{c} w_{2}+\left(w_{22}^{c}+w_{2}\right) w_{1}} x
$$

Under (24):

$$
c^{1}=\frac{(b+\rho) w_{11}^{c}\left(w_{2}+w_{21}^{c}\right)}{\left(w_{11}^{c}+w_{1}\right)\left(w_{22}^{c}+w_{2}+w_{21}^{c}\right)} x, \quad c^{2}=\frac{(b+\rho) w_{12}^{c}\left(w_{2}+w_{21}^{c}\right)}{\left(w_{11}^{c}+w_{1}\right)\left(w_{22}^{c}+w_{2}+w_{21}^{c}\right)} x
$$


where $w_{i l j}^{c}$ for all $i, j, l=1,2$ are assumed to be strictly positive. Let us note that the necessary condition establishes, as in the one-species specification, that the harvest rate of one player on species $j$, for $j=1,2$, is proportional to that of the other player.

Focusing our attention on the symmetric game, the following additional hypotheses over the parameters are considered:

$$
w_{1 i}=w_{2 i} \quad \text { for } i=1,2, \quad w_{1 i j}^{c}=w_{2 i j}^{c} \quad \text { for } i, j=1,2, \quad \rho_{1}=\rho_{2} .
$$

Let us note that under the symmetric hypothesis when the necessary condition for one player is satisfied it is also for the other player. As usual for symmetric games we look for symmetric solutions, i.e., the optimal harvest rates must satisfy $c^{1 i}=$ $c^{2 i}$ for $i=1,2$. Hence, if the set of optimal control is restricted to those symmetric, in the relationship between the optimal harvest rates of both players established in (25), necessary for a MPNE being a Pareto optimum, the following conditions have to be imposed:

$$
w_{12 i}^{c}=w_{11 i}^{c} \text { for } i=1,2 .
$$

Then, for the symmetric game the notation can be simplified as follows:

$$
w_{j i}=w_{i} \quad \text { for } i, j=1,2, \quad w_{i j l}^{c}=w_{l}^{c} \quad \text { for } i, j, l=1,2, \quad \rho_{1}=\rho_{2}=\rho .
$$

By replacing the relationships between the harvest rate of both players given by (25) in system (4), and substituting the costate vectors by

$$
\lambda^{i}=\mathrm{e}^{\rho_{i} t}\left(w_{1}^{c} / c^{i 1}, w_{2}^{c} / c^{i 2}\right)^{\top}, \quad i=1,2,
$$

where the necessary condition of maximization of each player's Hamiltonian (5) has been used, we obtain the following system of quasilinear partial differential equations:

$$
\begin{aligned}
& c_{t}^{11}+A\left(x_{1}, x_{2}, c^{11}\right) c_{x_{1}}^{11}+B\left(x_{1}, x_{2}, c^{12}\right) c_{x_{2}}^{11}=C\left(x_{1}, x_{2}, c^{11}, c^{12}\right), \\
& c_{t}^{12}+A\left(x_{1}, x_{2}, c^{11}\right) c_{x_{1}}^{12}+B\left(x_{1}, x_{2}, c^{12}\right) c_{x_{2}}^{12}=D\left(x_{1}, x_{2}, c^{11}, c^{12}\right),
\end{aligned}
$$

where

$$
\begin{aligned}
& A\left(x_{1}, x_{2}, c^{11}\right)=x_{1}\left(1-b_{11} \log x_{1}-b_{12} \log x_{2}\right)-2 c^{11}, \\
& B\left(x_{1}, x_{2}, c^{12}\right)=x_{2}\left(1-b_{21} \log x_{1}-b_{22} \log x_{2}\right)-2 c^{12}, \\
& C\left(x_{1}, x_{2}, c^{11}, c^{12}\right) \\
& \quad=c^{11}\left[-\rho+\frac{c^{11} w_{1}}{x_{1} w_{1}^{c}}+1-b_{11}-b_{11} \log x_{1}-b_{12} \log x_{2}-\frac{c^{11} w_{2}^{c} x_{2} b_{21}}{c^{12} w_{1}^{c} x_{1}}\right], \\
& D\left(x_{1}, x_{2}, c^{11}, c^{12}\right) \\
& \quad=c^{12}\left[-\rho+\frac{c^{12} w_{2}}{x_{2} w_{2}^{c}}+1-b_{22}-b_{21} \log x_{1}-b_{22} \log x_{2}-\frac{c^{12} w_{1}^{c} x_{1} b_{12}}{c^{11} w_{2}^{c} x_{2}}\right] .
\end{aligned}
$$

Since the problem is settled with an infinite time horizon stationary feedback equilibria are searched and then the time derivatives in system (26)-(27) are null. 
As the simplest case to find a solution of the system (26)-(27) we look for solutions in the class of linear solutions. That is, we want to find constants $A_{1}, B_{1}, A_{2}, B_{2}$ such that

$$
c^{11}=A_{1} x_{1}+B_{1} x_{2}, \quad c^{12}=A_{2} x_{1}+B_{2} x_{2},
$$

satisfy system (26)-(27).

After tedious computations the solutions summarized in Table 2 can be characterized as shown below.

- Two-species game, I. $B_{1}=0, A_{2}=0$.

In this case $c^{11}=A_{1} x_{1}, c^{12}=B_{2} x_{2}$, is an efficient subgame-perfect Nash equilibrium when constants $A_{1}$ and $B_{2}$ are given by

$$
A_{1}=\frac{\Upsilon w_{1}^{c}}{\Upsilon_{21}}, \quad B_{2}=\frac{\Upsilon w_{2}^{c}}{\Upsilon_{12}},
$$

where

$$
\begin{aligned}
& \Upsilon=b_{12} b_{21}-\left(b_{11}+\rho\right)\left(b_{22}+\rho\right), \\
& \Upsilon_{i j}=b_{i j}\left(w_{i}+2 w_{i}^{c}\right)-\left(b_{i i}+\rho\right)\left(w_{j}+2 w_{j}^{c}\right), \quad i, j=1,2, i \neq j .
\end{aligned}
$$

For the extraction rate being positive $A_{1}$ and $B_{2}$ have to be positive constants. This is guaranteed if all parameters $Y, Y_{i j}, i, j=1,2$ are either positive or negative. Furthermore, if the hypothesis $b_{21}=0$ is added, i.e., the evolution of the stock of the second species is independent of the stock of the first one, we obtain the following one-parametric family of efficient solutions:

$$
c^{11}=A_{1} x_{1}, \quad c^{12}=\frac{A_{1}\left(b_{22}+\rho\right) w_{2}^{c}}{A_{1}\left(w_{2}+2 w_{2}^{c}\right)-b_{12} w_{1}^{c}} x_{2} .
$$

Both harvest rates are positive if the following conditions are satisfied:

$$
A_{1}>0, \quad A_{1}>\frac{b_{12} w_{1}^{c}}{w_{2}+2 w_{2}^{c}} .
$$

It can be proved that for this kind of solutions the equilibrium state trajectory presents different types of stability. First, it converges globally to a unique steady state if $b_{11} b_{22}-b_{12} b_{21}>0$ and $b_{11}+b_{22}>0$. Second, if $b_{11} b_{22}-b_{12} b_{21}>0$ and $b_{11}+b_{22}=0$ the equilibrium trajectory follows a closed curve, i.e., the steady state behaves as a center. Third, if $b_{11} b_{22}-b_{12} b_{21}<0$ the steady state is a saddle point, guaranteeing that the equilibrium state trajectory converges asymptotically to the steady state. These results are in concordance with those established in Clemhout and Wan (1985) in the framework of a predator-prey system.

- Two-species game, II. $B_{1} \neq 0, A_{2} \neq 0, b_{11}=b_{21}, b_{12}=b_{22}$.

Under these hypotheses several cases appear, each of them leading to a oneparametric family of efficient MPNE. 
Table 2

Example 1. Two species symmetric game

Conditions Symmetric Markov perfect Nash equilibria: $c^{1 i}=c^{2 i}, i=1,2$

Linear Strategies: $c^{11}=A_{1} x_{1}+B_{1} x_{2}, c^{12}=A_{2} x_{1}+B_{2} x_{2}$

$\forall i, j=1,2$

$B_{1}=0, A_{2}=0, A_{1}=\frac{\Upsilon w_{1}^{c}}{\Upsilon_{21}}, B_{2}=\frac{\Upsilon w_{2}^{c}}{\Upsilon_{12}}$

$\Upsilon, Y_{i j}>0$

or

$\Upsilon, \Upsilon_{i j}<0$

Under (28)

$B_{1}=0, A_{2}=0, b_{21}=0, B_{2}=\frac{A_{1}\left(b_{22}+\rho\right) w_{2}^{c}}{A_{1}\left(w_{2}+2 w_{2}^{c}\right) \quad b_{12} w_{1}^{c}}$ for all $A_{1}>0$.

Under (Aa)

$B_{1} \neq 0, A_{2} \neq 0, b_{11}=b_{21}>0, b_{12}=b_{22}>0, A_{1}=\frac{b_{22} w_{1}^{c}}{w_{2}}, B_{2}=\frac{b_{11} w_{2}^{c}}{w_{1}}$,

or $(\mathrm{Ba})$ and

$w_{1} \neq 0, w_{2} \neq 0, A_{2}=\frac{b_{11} w_{1}^{c} \Lambda_{1} w_{2}^{c}}{B_{1} w_{1}^{2}\left(w_{1}+2 w_{1}^{c}\right)}$ for all $B_{1}>0$.

$\Lambda_{1}>0$

Under $(\mathrm{Ab})$

$B_{1} \neq 0, A_{2} \neq 0, b_{11}=b_{21}>0, b_{12}=b_{22}>0, A_{1}=\frac{b_{22} w_{1}^{c}}{w_{2}}, B_{2}=\frac{b_{11} w_{2}^{c}}{w_{1}}$,

or $(\mathrm{Bb})$ and

$w_{1} \neq 0, w_{2} \neq 0, A_{2}=\frac{b_{11} w_{1}^{c} \Delta_{1} w_{2}^{c}}{B_{1} w_{1}^{4}}$ for all $B_{1}>0$.

$\Delta_{1}>0$

Under(29)

$B_{1} \neq 0, A_{2} \neq 0, b_{11}=b_{21}>0, b_{12}=b_{22}, A_{1}=\frac{\Xi}{2 w_{1}}, B_{2}=\frac{b_{11} w_{2}^{c}}{w_{1}}$,

and $\Xi>0$

$w_{1} \neq 0, B_{1}=\frac{b_{11} w_{2}^{c} \Xi}{2 A_{2} w_{1}^{2}}$ for all $A_{2}>0$.

Under (30)

$B_{1} \neq 0, A_{2} \neq 0, b_{11}=b_{21}=0, b_{12}=b_{22}>0, A_{1}=\frac{\Theta}{2 w_{2}^{2}}, B_{2}=\frac{2 b_{22} w_{1}^{c} w_{2}^{c}}{w_{2}^{2}}$,

and $\Theta>0$

$w_{1}=0, w_{2} \neq 0, B_{1}=\frac{b_{22} w_{1}^{c} w_{2}^{c} \Theta}{A_{2} w_{2}^{4}}$ for all $A_{2}>0$.

$B_{1} \neq 0, A_{2} \neq 0, b_{11}=b_{21}=0, b_{12}=b_{22}, w_{1}=0, A_{1}=\frac{\rho 2 B_{2}}{2}$,

$B_{1}=\frac{B_{2}\left(\rho 2 B_{2}\right)}{2 A_{2}}$ for all $A_{2}>0$ and $0<B_{2}<\rho / 2$.

$b_{12}=b_{22}=0, A_{2}=A_{1}=0, B_{2}=\rho / 2$, for all $B_{1}>0$.

1. $A_{1}=\frac{b_{22} w_{1}^{c}}{w_{2}}, B_{2}=\frac{b_{11} w_{2}^{c}}{w_{1}}, w_{1} \neq 0, w_{2} \neq 0$.

(a) Choosing

$$
A_{2}=\frac{b_{11} w_{1}^{c} \Lambda_{1} w_{2}^{c}}{B_{1} w_{1}^{2}\left(w_{1}+2 w_{1}^{c}\right)}, \text { for all } B_{1}>0
$$


where

$$
\Lambda_{i}=\left(\rho+b_{i i}\right) w_{i}-2 b_{i i} w_{j}^{c}, i, j=1,2, i \neq j,
$$

generates a family of efficient Nash Equilibria, if one (or both) of the following conditions on the parameters is satisfied:

(Aa)

$$
\begin{aligned}
& \Lambda_{i} w_{j}-b_{j j} w_{i}\left(w_{i}+2 w_{i}^{c}\right)=0, i, j=1,2, i \neq j, \\
& b_{22}=1 .
\end{aligned}
$$

(Ba)

$$
\begin{aligned}
& 4 b_{22} w_{1}^{c}\left(w_{2}+w_{2}^{c}\right)-\left(b_{22}+\rho\right) w_{2}^{2}=0, \\
& b_{11} w_{2}^{2}-2 b_{22} w_{1} w_{1}^{c}=0, \\
& \Lambda_{1} w_{2}-2 b_{22} w_{1}\left(w_{1}+2 w_{1}^{c}\right)=0 .
\end{aligned}
$$

(b) Choosing

$$
A_{2}=\frac{b_{11} w_{1}^{c} w_{2}^{c} \Delta_{1}}{B_{1} w_{1}^{4}}, \text { for all } B_{1}>0,
$$

where

$$
\Delta_{i}=\left(\rho+b_{i i}\right) w_{i}^{2}-2 b_{i i}\left(w_{i}+2 w_{i}^{c}\right) w_{j}^{c}, i, j=1,2, i \neq j,
$$

generates a family of efficient Nash equilibria if one (or both) of the following conditions on the parameters is satisfied:

$(\mathrm{Ab})$

$$
\begin{aligned}
& \Lambda_{2} w_{1}-b_{11} w_{2}\left(w_{2}+2 w_{2}^{c}\right)=0, \\
& b_{22} w_{1}^{2}-2 b_{11} w_{2} w_{2}^{c}=0, \\
& w_{2} \Delta_{1}-b_{22} w_{1}^{3}=0 .
\end{aligned}
$$

$(\mathrm{Bb})$

$$
\begin{aligned}
& b_{i i} w_{j}^{2}-2 b_{j j} w_{i} w_{i}^{c}=0, i, j=1,2, i \neq j, \\
& w_{1} b_{11} w_{2}^{c} \Delta_{2}-w_{2} b_{22} w_{1}^{c} \Delta_{1}=0 .
\end{aligned}
$$

To guarantee positive values of constants $A_{1}, B_{2}$ and $A_{2}$ is sufficient to choose $b_{22}>0, b_{11}>0$ and $\Lambda_{1}>0$ or $\Delta_{1}>0$, for the first and second case, respectively. 2. $A_{1}=\frac{\Xi}{2 w_{1}}, B_{2}=\frac{b_{11} w_{2}^{c}}{w_{1}}, w_{1} \neq 0$, where $\Xi=\rho w_{1}-2 b_{11} w_{2}^{c}$.

Choosing

$$
B_{1}=\frac{b_{11} w_{2}^{c} \Xi}{2 A_{2} w_{1}^{2}}, \text { for all } A_{2}>0,
$$

generates a family of efficient Nash equilibria, if the following condition on the parameters is satisfied:

$$
2 b_{11}\left(w_{1}^{c}+w_{2}^{c}\right)-\rho w_{1}=0 .
$$


Positive values of constants $A_{1}, B_{2}$ and $B_{1}$ are guaranteed when $b_{11}>0$ and $\Xi>0$.

3. $w_{1}=0, b_{11}=b_{21}=0$.

(a) $A_{1}=\frac{\Theta}{2 w_{2}^{2}}, B_{2}=\frac{2 b_{22} w_{1}^{c} w_{2}^{c}}{w_{2}^{2}}, w_{2} \neq 0$, where $\Theta=\rho w_{2}^{2}-4 b_{22} w_{1}^{c} w_{2}^{c}$.

Choosing

$$
B_{1}=\frac{b_{22} w_{1}^{c} w_{2}^{c} \Theta}{A_{2} w_{2}^{4}}, \text { for all } A_{2}>0,
$$

generates a family of efficient Nash equilibria, if the following condition is satisfied:

$$
w_{2}-2 w_{1}^{c}=0 .
$$

The fulfillment of inequalities $b_{22}>0$ and $\rho w_{2}^{2}-4 b_{22} w_{1}^{c} w_{2}^{c}>0$ implies positive values of constants $A_{1}, B_{2}$ and $B_{1}$.

(b) $A_{1}=\frac{\rho 2 B_{2}}{2}, B_{1}=\frac{B_{2}\left(\rho 2 B_{2}\right)}{2 A_{2}}, A_{2} \neq 0$.

For all $A_{2}>0$ and $0<B_{2}<\rho / 2$ we have constants $A_{1}$ and $B_{1}$ taking positive values and leading to a family of efficient MPNE.

Under the hypothesis $b_{11}=b_{21}, b_{12}=b_{22}$ being $B_{1} \neq 0, A_{2} \neq 0$, it can be proved that the equilibrium state trajectory converges asymptotically to the steady state if and only if $b_{11}+b_{22}>0$. In this case, the stability analysis shows that nor global stability of the equilibrium trajectory neither a closed curve around the steady state can appear.

- Two-species game, III. $b_{12}=b_{22}=0$.

Choosing $A_{2}=A_{1}=0, B_{2}=\rho / 2$ and any positive value for $B_{1}$, we obtain a MPNE being Pareto optimum.

The concavity of $H^{i}$ with respect to his or her own control variables is ensured. As we pointed out in Remark 4, this assures that the necessary condition (11) for the $i$ th player is sufficient too. Moreover, the Hamiltonians $H^{1}$ and $H^{2}$ are strictly concave with respect to all the control variables and knowing that the symmetric game condition (11) is satisfied for both players, then all the MPNE we have characterized for this example correspond to absolute cooperative solutions.

Example 2. Following Hämäläinen et al. (1985) the following two-person twospecies differential game is considered:

$$
\begin{aligned}
& \max _{c^{i j}} \int_{0}^{T} \mathrm{e}^{\rho_{i} t}\left[\sum_{j=1}^{2}\left(\pi_{j} c^{i j}-C_{i j}\left(c^{i j}, x_{j}\right)\right)\right] \mathrm{d} t, \\
& \text { s.t. } \dot{x}_{j}=F_{j}\left(x_{j}\right)-\sigma\left(x_{j}-x_{k}\right)-c^{1 j}-c^{2 j},
\end{aligned}
$$

$i, j=1,2 ; k=1,2 ; k \neq j$.

This model corresponds to a game of exploitation of the so-called transboundary fisheries. As in the previous example state variables $x_{1}$ and $x_{2}$ represent the stock of the first and second species, and $c^{i j}$ denotes the harvest rate of each country $i$ with 
respect to species $j$. The model can be interpreted as one of the two fisheries with the same kind of fish in two different locations. Function $\sigma\left(x_{j}-x_{k}\right)$ corresponds to a diffusion factor that represents the natural transfer of fish from one location to the other. $\pi_{j}$ is the price of fish at fishery $j, C_{i j}(\cdot, \cdot)$ is the cost function for using the harvest rate $c^{i j}$ at stock level $x_{j}$. Finally, $\rho_{i}$ is the discount rate used by country $i$.

In what follows, the cost functions are assumed to be quadratic

$$
C_{i j}\left(c^{i j}, x_{j}\right)=\frac{1}{2}\left(\alpha_{i j}\left(c^{i j}\right)^{2}+\beta_{i j} x_{j}^{2}+2 \gamma_{i j} x_{j} c^{i j}\right), \quad i, j=1,2,
$$

with $\alpha_{i j}>0, \beta_{i j}>0, \gamma_{i j}<0$. We will illustrate our results for different specifications of the renovation functions, $F_{j}\left(x_{j}\right)$.

The necessary condition (11) for a MPNE being a Pareto optimum for the second player reads:

$$
c^{2 i}=\frac{\pi_{i}-\gamma_{2 i} x_{i}}{\alpha_{2 i}}, \quad i=1,2 .
$$

Since $\gamma_{i j}<0$ for all $i, j=1,2$, the extraction rates for the second player always take positive values. It is worth noting that for this example the necessary condition for the efficiency of the MPNE does not establish a relationship between the control variables of the two players, as in the previous example. On the contrary, (31) directly gives the functional expressions for the two control variables for the second player.

By replacing the expressions given by (31) in the equations of system corresponding to the second player, and substituting the costate vectors by

$$
\lambda^{i}=\mathrm{e}^{\rho_{i} t}\left(\pi_{1}-\alpha_{i 1} c^{i 1}-\gamma_{i 1} x_{1}, \pi_{2}-\alpha_{i 2} c^{i 2}-\gamma_{i 2} x_{2}\right)^{\top}, \quad i=1,2,
$$

where the necessary condition of maximization of each player's Hamiltonian (5) has been used, the following pair of equations is obtained:

$$
3 \gamma_{2 i}\left[F_{i}\left(x_{i}\right)-\sigma\left(x_{i}-x_{j}\right)-c^{1 i}-\frac{\pi_{i}-\gamma_{2 i} x_{i}}{\alpha_{2 i}}\right]-\beta_{2 i} x_{i}-\gamma_{2 i}\left(\frac{\pi_{i}-\gamma_{2 i} x_{i}}{\alpha_{2 i}}\right)=0,
$$

for $i, j=1,2, i \neq j$. From these equations one can obtain the expressions for the control variables of the first player:

$$
c^{1 i}=F_{i}\left(x_{i}\right)-\left(\sigma-\frac{4}{3} \frac{\gamma_{2 i}}{\alpha_{2 i}}+\frac{1}{3} \frac{\beta_{2 i}}{\gamma_{2 i}}\right) x_{i}+\sigma x_{j}-\frac{4}{3} \frac{\pi_{i}}{\alpha_{2 i}}, \quad i, j=1,2, i \neq j,
$$

where $\gamma_{21}$ and $\gamma_{22}$ are assumed to be not null, since $\gamma_{21}=\gamma_{22}=0$ implies $\beta_{21}=$ $\beta_{22}=0$, which contradicts the hypothesis $\beta_{i j}>0$, for all $i, j=1,2$. To guarantee that the harvest rates of the first player are positive, conditions on the parameters of the model which depend on the renovation functions, $F_{i}(\cdot), i=1,2$ have to be imposed.

Our aim now is to prove when the harvest rates for the first player, given by (32), correspond to a solution to the pair of quasilinear partial differential equations of system (4) associated to the first player, where as before the harvest rates of the second player given by (31) and the costate vectors have been 
replaced

$$
\begin{aligned}
& c_{t}^{11}+A\left(x_{1}, x_{2}, c^{11}\right) c_{x_{1}}^{11}+B\left(x_{1}, x_{2}, c^{12}\right) c_{x_{2}}^{11}=C\left(x_{1}, x_{2}, c^{11}, c^{12}\right), \\
& c_{t}^{12}+A\left(x_{1}, x_{2}, c^{11}\right) c_{x_{1}}^{12}+D\left(x_{1}, x_{2}, c^{12}\right) c_{x_{2}}^{12}=E\left(x_{1}, x_{2}, c^{11}, c^{12}\right),
\end{aligned}
$$

where

$$
\begin{gathered}
A\left(x_{1}, x_{2}, c^{11}\right)=F_{1}\left(x_{1}\right)-\sigma\left(x_{1}-x_{2}\right)-c^{11}-\frac{\pi_{1}-\gamma_{21} x_{1}}{\alpha_{21}}, \\
B\left(x_{1}, x_{2}, c^{12}\right)=\left(\alpha_{11}\right)^{1}\left[F_{2}\left(x_{2}\right)-\sigma\left(x_{2}-x_{1}\right)-c^{12}-\frac{\pi_{2}-\gamma_{22} x_{2}}{\alpha_{22}}\right], \\
C\left(x_{1}, x_{2}, c^{11}, c^{12}\right)=\left(\alpha_{11}\right)^{1}\left[\left(\pi_{1}-\alpha_{11} c^{11}-\gamma_{11} x_{1}\right)\left(\rho_{1}+F_{1}^{\prime}\left(x_{1}\right)-\sigma+\frac{\gamma_{21}}{\alpha_{21}}\right)\right. \\
+\left(\pi_{2}-\alpha_{12} c^{12}-\gamma_{12} x_{2}\right) \sigma-\beta_{11} x_{1}-\gamma_{11} c^{11} \\
\left.+\gamma_{11}\left(F_{1}\left(x_{1}\right)-\sigma\left(x_{1}-x_{2}\right)-c^{11}-\frac{\pi_{1}-\gamma_{21} x_{1}}{\alpha_{21}}\right)\right], \\
\begin{aligned}
&\left.\alpha_{1}, x_{2}, c^{12}\right)=\left(\alpha_{12}\right)^{1}\left[F_{2}\left(x_{2}\right)-\sigma\left(x_{2}-x_{1}\right)-c^{12}-\frac{\pi_{2}-\gamma_{22} x_{2}}{\alpha_{22}}\right], \\
& E\left(x_{1}, x_{2}, c^{11}, c^{12}\right)=\left(\alpha_{12}\right){ }^{1}\left[\left(\pi_{2}-\alpha_{12} c^{12}-\gamma_{12} x_{2}\right)\left(\rho_{1}+F_{2}^{\prime}\left(x_{2}\right)-\sigma+\frac{\gamma_{22}}{\alpha_{22}}\right)\right. \\
&+\left(\pi_{1}-\alpha_{11} c^{11}-\gamma_{11} x_{1}\right) \sigma-\beta_{12} x_{2}-\gamma_{12} c^{12} \\
&+\left.\gamma_{12}\left(F_{2}\left(x_{2}\right)-\sigma\left(x_{2}-x_{1}\right)-c^{12}-\frac{\pi_{2}-\gamma_{22} x_{2}}{\alpha_{22}}\right)\right] .
\end{aligned}
\end{gathered}
$$

Assuming an infinite horizon game, we search for stationary feedback equilibria. Then the time dependency in system (33)-(34) can be eliminated. Replacing the harvest rates for the first player given by (32) in (33) and (34), we get the following pair of equations that have to be satisfied for an efficient MPNE:

$$
\begin{aligned}
& {\left[\pi_{i}-\alpha_{1 i}\left(F_{i}\left(x_{i}\right)-\sigma\left(x_{i}-x_{j}\right)\right)-\gamma_{1 i} x_{i}\right]\left[\rho_{1}+F_{i}^{\prime}\left(x_{i}\right)-\sigma+\frac{\gamma_{2 i}}{\alpha_{2 i}}\right]} \\
& -\left[\beta_{1 i} x_{i}+\gamma_{1 i}\left(F_{i}\left(x_{i}\right)-\sigma\left(x_{i}-x_{j}\right)\right)\right] \\
& +\left[\pi_{j}-\alpha_{1 j}\left(F_{j}\left(x_{j}\right)-\sigma\left(x_{j}-x_{i}\right)\right)-\gamma_{1 j} x_{j}\right] \sigma \\
& +\frac{\pi_{i}-\gamma_{2 i} x_{i}}{3 \alpha_{2 i}}\left[\alpha_{1 i}\left(4 \rho_{1}-3 \sigma+3 F_{i}^{\prime}\left(x_{i}\right)+\frac{8 \gamma_{2 i}}{3 \alpha_{2 i}}+\frac{\beta_{2 i}}{3 \gamma_{2 i}}\right)+5 \gamma_{1 i}\right] \\
& +\frac{\beta_{2 i}}{3 \gamma_{2 i}} x_{i}\left[2 \gamma_{1 i}+\alpha_{1 i}\left(\rho_{1}-\frac{\gamma_{2 i}}{3 \alpha_{2 i}}+\frac{\beta_{2 i}}{3 \gamma_{2 i}}\right)\right] \\
& +\frac{\beta_{2 j}}{3 \gamma_{2 j}} x_{j}\left(\alpha_{1 j}-\alpha_{1 i}\right) \sigma+\frac{\pi_{j}-\gamma_{2 j} x_{j}}{3 \alpha_{2 j}}\left(4 \alpha_{1 j}-\alpha_{1 i}\right) \sigma=0, \\
& \quad i, j=1,2, i \neq j .
\end{aligned}
$$


We search for conditions over the parameters that guarantee the fulfillment of the above equations. First, we assume that the renovation functions are described by logistic functions, i.e., $F_{i}\left(x_{i}\right)=r_{i} x_{i}\left(1-x_{i} / K_{i}\right), i=1,2$, where $r_{i}$ and $K_{i}$ are the intrinsic growth rate and the carrying capacity, respectively, and which are assumed to be strictly positive. For this specification both equations are third degree polynomials in the variables $x_{1}$ and $x_{2}$. All the coefficients of the polynomials are zero if and only if $r_{1}=r_{2}=0$. Since $r_{i}, i=1,2$ are assumed strictly positive, we can conclude that if the renovation functions follow logistic expressions, there is no efficient MPNE.

Second, we assume that the renovation functions are linear, that is, $F_{i}\left(x_{i}\right)=$ $r_{i} x_{i}, i=1,2$. In this case both equations are first degree polynomials in the variables $x_{1}$ and $x_{2}$. Making the three coefficients of each polynomial (independent term, $x_{1}$ and $x_{2}$ ) null leads to six equations that involve the nineteen parameters of the model. Because the complexity of the equations and because the number of parameters are large, checking if the cooperative solution is a Nash equilibrium is not a straightforward task. However, since the number of parameters is much greater than the number of equations it is not difficult to find a set of values of the parameters satisfying all of them. For example, a set of parameters associated with an efficient MPNE can be found, fixing $\pi_{1}=\pi_{2}, \sigma=0, \alpha_{i j}=1, \gamma_{i j}=-1$ for $i, j=1,2$, and choosing

$$
\begin{aligned}
& r_{1}=4-r_{2}-\rho_{1}, \quad r_{2}=\frac{1}{8}\left(32+\beta_{22}-21 \rho\right), \quad \beta_{21}=8-\beta_{22}+24 \rho_{1}, \\
& \beta_{12}=\frac{1}{324}\left[128+35 \beta_{22}^{2}+12 \rho_{1}-63 \rho_{1}^{2}-4 \beta_{22}\left(-38+21 \rho_{1}\right)\right], \\
& \beta_{11}=\frac{1}{324}\left[3584+35 \beta_{22}^{2}+16428 \rho_{1}^{2}-4 \beta_{22}\left(178+399 \rho_{1}\right)\right], \\
& 0<\beta_{22}<13 \rho_{1}, \quad \rho_{1} \in(0,0.911949) .
\end{aligned}
$$

The last two inequalities ensure that parameters $r_{i}$ and $\beta_{i j}$ are positive. Positive values for the stationary state leading to an equilibrium state trajectory which converges globally to the unique steady are guaranteed if one of the following conditions on the parameters is satisfied:

(A) $\rho_{1}<0.2759$

$$
\begin{gathered}
\frac{1}{3}\left(87 \rho_{1}-36+15 r_{2}\right)<\beta_{22}<27 \rho_{1}+3 r_{2}-5, \\
\frac{1}{15}\left(39-87 \rho_{1}\right)<r_{2}<\frac{1}{12}\left(36-87 \rho_{1}\right),
\end{gathered}
$$

(B) $\rho_{1}>0.2759$

$$
1<\beta_{22}<27 \rho_{1}+3 r_{2}-5, \quad \frac{1}{3}\left(6-27 \rho_{1}\right)<r_{2}<\frac{1}{12}\left(36-87 \rho_{1}\right) .
$$

With this choice of the parameters, and since the concavity of the Hamiltonian of the second player with respect to his or her own control variables can be proved, the 
necessary condition (11) for this player is sufficient too, as we pointed out in Remark 4. Then, we have found a MPNE, given by the expressions (32) and (31), which qualifies as a Pareto optimum. Let us note that in this example, the efficient MPNE is not an absolute cooperative solution, since the necessary condition (11) is not fulfilled for the first player. In fact, for the symmetric game it has been proved that choosing linear renovation functions and looking for linear strategies there are no solutions satisfying the necessary conditions for both players simultaneously. When the renovation functions follow a logistic the same result applies if linear or quadratic strategies are considered.

\section{Conclusions}

We have derived necessary and sufficient conditions to characterize MPNE being Pareto efficient. The proposed method is based on the characterization of Markov Nash equilibria as solutions to a system of quasilinear partial differential equations. Our approach also uses a compatibility condition that makes it possible to verify the existence of common solutions of several partial differential equations. For differential games with unidimensional state and control variables, the nonfulfillment of this compatibility condition provides a negative criterium based on a full computational method, establishing that the Pareto optimum cannot be a Nash equilibrium.

Even though the efficiency of Nash equilibria is not a common property, it is a very desirable property, since it makes the efficient solution self-enforcing. We have proved that there are interesting economic differential games, in particular, the dynamic fishery games, that present this property, although they are non-cooperative in its mode of play. We have also proved that it can be the case that the cooperative solution can be achieved as an open-loop Nash equilibrium of a non-cooperative game, but not as a MPNE.

\section{Acknowledgements}

We are grateful to the editor Kenneth L. Judd and an anonymous referee for helpful comments. The research of the first author was supported by MCYT under project BEC2002-02361 and JCYL under project VA51/03, cofinanced by FEDER funds. The research of the second author was supported by MCYT under project BFM2002-00425 and JCYL under project VA099/04 cofinanced by FEDER funds.

\section{References}

Ames, W.F., 1965. Nonlinear Partial Differential Equations in Engineering. Academic Press, New York. Chiarella, C., Kemp, M.C., Van Long, N., 1984. On the economics of international fisheries. International Economic Review 25, 8592. 
Clemhout, S., Wan, H.Y., 1985. Dynamic common property resources and environmental problems. Journal of Optimization Theory and Applications 46, 471481.

Dockner, E., Jørgensen, S., Van Long, N., Sorger, G., 2000. Differential Games in Economics and Management. Cambridge University Press, Cambridge.

Dubey, P., 1986. Inefficiency of Nash equilibria. Mathematics of Operations Research 11, 18.

Ehtamo, H., Hamalainen, R.P., 1989. Incentive strategies and equilibria for dynamic games with delayed information. Journal of Optimization Theory and Applications 63, 355369.

Ehtamo, H., Hamalainen, R.P., 1993. A cooperative incentive equilibrium for a resource management problem. Journal of Economic Dynamics and Control 17, 659678.

Hamalainen, R.P., Haurie, A., Kaitala, V., 1985. Equilibria and threats in a fishery management game. Optimal Control Applications and Methods 6, 315333.

Haurie, A., Pohjola, M., 1987. Efficient equilibria in a differential game of capitalism. Journal of Economic Dynamics and Control 11, 6578.

Jørgensen, S., Zaccour, G., 2001. Incentive equilibrium strategies and welfare allocation in a dynamic game of pollution control. Automatica 37, 2936.

Leitmann, G., 1974. Cooperative and Noncooperative Many Players Differential Games. Springer, New York.

Martín Herrán, G., Rincón Zapatero, J.P., 2002. Computation of Markov perfect Nash equilibria without Hamilton Jacobi Bellman equations. In: Zaccour, G. (Ed.), Optimal Control and Differential Games. Essays in Honor of Steffen Jørgensen. Advances in Computational Management Science. Kluwer Academic Publishers, Boston, pp. 135151.

Rincón Zapatero, J.P., 2004. Characterization of Markovian equilibria in a class of differential games. Journal of Economic Dynamics and Control 28, 12431266.

Rincón Zapatero, J.P., Martínez, J., Martín Herrán, G., 1998. New method to characterize subgame perfect Nash equilibria in differential games. Journal of Optimization Theory and Applications 96, 377395.

Rincón Zapatero, J.P., Martín Herrán, G., Martínez, J., 2000. Identification of efficient subgame perfect Nash equilibria in a class of differential games. Journal of Optimization Theory and Applications 104, 235242.

Starr, A.W., Ho, Y.C., 1969. Further properties of nonzero sum differential games. Journal of Optimization Theory and Applications 3, 207219.

Tolwinski, B., Haurie, A., Leitmann, G., 1986. Cooperative equilibria in differential games. Journal of Mathematical Analysis and Applications 119, 192202. 